\title{
Top quark production measurements using the ATLAS detector at the LHC
}

\author{
Naim Bora Atlay on behalf of the ATLAS Collaboration ${ }^{1, a}$ \\ ${ }^{1}$ University of Siegen \\ Walter-Flex Str. 3, 57072, Siegen, Germany
}

\begin{abstract}
A selection from the inclusive top-quark pair production and single top-quark production cross-section measurements in proton-proton collisions with the ATLAS detector at the Large Hadron Collider is presented, with data collected in 2012 and early 2015 at centre-of-mass energies of $8 \mathrm{TeV}$ and $13 \mathrm{TeV}$, respectively.
\end{abstract}

\section{Introduction}

The top-quark, discovered over twenty years ago [1,2], is the heaviest known fundamental particle and close to the scale of electroweak symmetry breaking. Having a lifetime at the order of $10^{-25} \mathrm{~s}$, the top-quark decays before it hadronises which enables the decay products to carry its "bare quark" properties. The study of its production and decay properties forms a core part of the LHC physics programme. Due to the high amount of top-quarks the LHC can produce, the ATLAS experiment [3] is advantageous in comparison to former experiments. The LHC, being a proton-proton collider, the top-quark production is dominated by gluon-gluon fusion process.

\section{Top-quark pair production}

At the LHC, top quarks can be produced in either quark-antiquark pairs $(t \bar{t})$ or as single quarks, where the former is the primary production. In this section, three different measurements of the top-quark pair production are presented.

\subsection{Measurement of the $t \bar{t}$ production cross-section using $e \mu$ events with $b$-tagged jets}

This measurement [4] uses a data sample of $3.2 \mathrm{fb}^{-1}$ of proton-proton collisions at a centre-of-mass energy of $\sqrt{s}=13 \mathrm{TeV}$ and is performed using dileptonic events with an opposite-charge electronmuon pair in the final state. The algorithm used to tag jets containing $b$-quarks exploits track impact parameters and reconstructed secondary vertices. The $t \bar{t}$ cross-section is extracted by counting the $e \mu$ events with exactly one and exactly two b-tagged jets. The cross-section is extracted simultaneously with the combined probability to reconstruct and tag a b-jet from the top-quark decay, which minimises the associated systematic uncertainties. The comparison between data and simulations for

ae-mail: naim.bora.atlay@cern.ch 


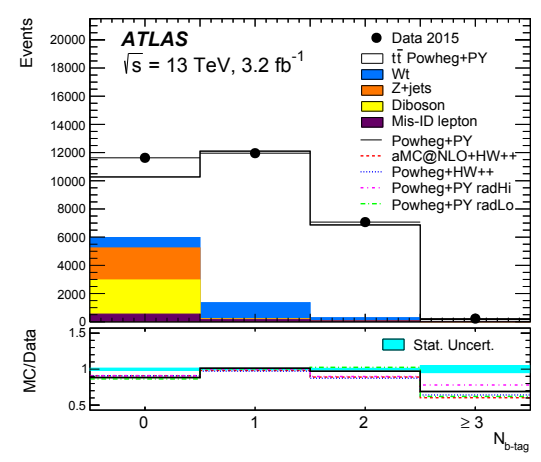

Figure 1: Distribution of the number of b-tagged jets. The data is compared to the expectation from signal and background simulations. The lower part of the figure shows the ratio of simulation to data, using various tt signal samples, and the cyan band indicates the statistical uncertainty [4].

signal and backgrounds in terms of the number of $b$-tagged jets is shown in figure 1 . The extracted cross-section [4] is

$$
\left.\sigma_{t \bar{t}}=818 \pm 8(\text { stat. }) \pm 27 \text { (syst. }\right) \pm 19(\text { lumi }) \pm 12 \text { (beam) } \mathrm{pb},
$$

where the theoretical prediction to be compared with is $\sigma_{t \bar{t}}=832_{46}^{40} \mathrm{pb}$ [4].

\subsection{Measurements of top-quark pair differential cross-sections in the lepton+jets channel}

Differential cross-section measurements of top-quark pair production with respect to different kinematic variables are important to study the characteristics of $t \bar{t}$ production. In addition, effects beyond the Standard Model can be observed as deviations of differential distributions with respect to the Standard Model predictions.

With this motivation, a set of measurements [5] of the $t \bar{t}$ production cross-section as a function of different properties of the reconstructed top quark and of the $t \bar{t}$ system has been performed. The results are unfolded both to a fiducial particle-level phase space and to the full phase space. Unfolding to a fiducial particle-level phase space and using variables directly related to detector observables allows precision tests of QCD by avoiding large model-dependent extrapolation corrections to the parton-level top-quark and to a phase space region outside the detector sensitivity.

The measurement is done using a data sample of $20.3 \mathrm{fb}^{-1}$ collected at a centre-of-mass energy of $\sqrt{s}=8 \mathrm{TeV}$. The baseline observables to perform the differential measurements are the transverse momentum $\left(p_{T}^{t, h a d}\right)$, the absolute value of the rapidity $\left(\left|y^{t, h a d}\right|\right)$ of the hadronically decaying top-quark, and the transverse momentum $\left(p_{T}^{t \bar{t}}\right)$, the absolute value of the rapidity $\left|y^{t \bar{t}}\right|$ and the invariant mass $m^{t \bar{t}}$ of the $t \bar{t}$ system. The observables have been chosen to emphasise the $t \bar{t}$ production process. The observables are also sensitive to effects of initial and final-state radiation, to the different parton distribution functions, and to non-resonant processes and higher-order corrections. The measurement uses events in the lepton+jets channel, requiring exactly one charged lepton and at least four jets with at least two jets tagged as originating from a $b$-quark. Distributions of two of the baseline 

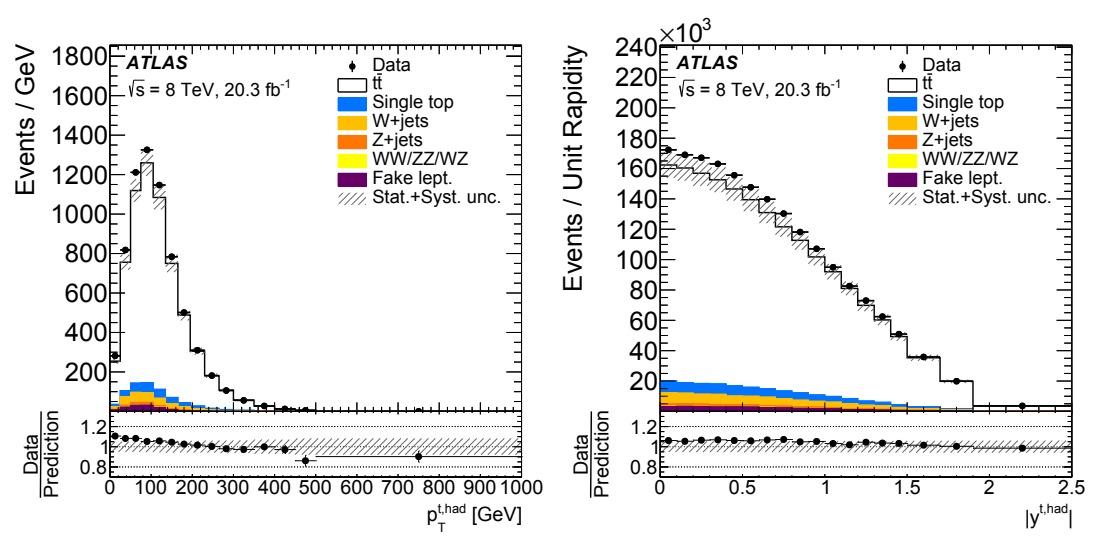

Figure 2: Distributions of observables of the combined electron and muon selections at detector level: hadronic top-quark transverse momentum, $p_{T}^{t}$, (left) and absolute value of the rapidity, $\left|y^{t, h a d}\right|$, (right). Data distributions are compared to predictions, using Powheg+PYTHIA as the $t \bar{t}$ signal model. The lower part of the figure shows the ratio data to simulation. The hashed area indicates the combined statistical and systematic uncertainties on the total prediction, excluding systematic uncertainties related to the modelling of the $t \bar{t}$ system [5].

observables for the combined electron and muon selections at detector level are shown in figure 2 . A fair agreement between the measurement and the predictions over a wide kinematic range is to be found. However, most generators predict a harder top-quark transverse momentum distribution at high values than what is observed in the data. The agreement of the data with the prediction for this observable improves, when the NNLO corrections are taken into account. Distributions for the full phase-space normalised differential cross-sections as a function of the transverse momentum, $p_{T}^{t \bar{t}}$ and for absolute value of the rapidity, $\left|y^{\text {thad }}\right|$ are shown in figure 3 .

\subsection{Measurement of the top-quark production cross-sections in association with a massive vector-boson in multi-lepton final states}

The rate of top-quark pair production in association with a massive vector boson(W or Z boson), $t \bar{t} Z$ and $t \bar{t} W$, could be altered in the presence of physics beyond the Standard Model, such as vectorlike quarks, strongly coupled Higgs bosons or technicolor. The cross-section measurements [6] of $t \bar{t} Z$ and $t \bar{t} W$ play therefore an important role for the validity check of the Standard Model at this new energy regime. In addition, a measurement of $\sigma_{t \bar{t} Z}$ allows to extract information on the neutral current coupling of the top-quark. The measurements of $\sigma_{t \bar{t} Z}$ and $\sigma_{t \bar{t} W}$ are performed using $3.2 \mathrm{fb}^{-1} \mathrm{of}$ proton-proton collision data at $\sqrt{s}=13 \mathrm{TeV}$. Depending on the decay modes of the top-quarks, $\mathrm{W}$, and $\mathrm{Z}$ bosons, the final state can include between zero and four isolated, prompt leptons. The measurement considers final states with two same-sign charged muons, or three and four leptons. The analysis channels and the targeted decay modes of the $t \bar{t} Z$ and $t \bar{t} W$ processes are presented in table 1.

\section{Same-sign dimuon analysis}

This region targets the $t \bar{t} W$ process and since electrons have a much larger charge misidentification probability, inducing a significant background from top-quark pairs, this region has the highest sensitivity among all same sign dilepton regions [7]. 

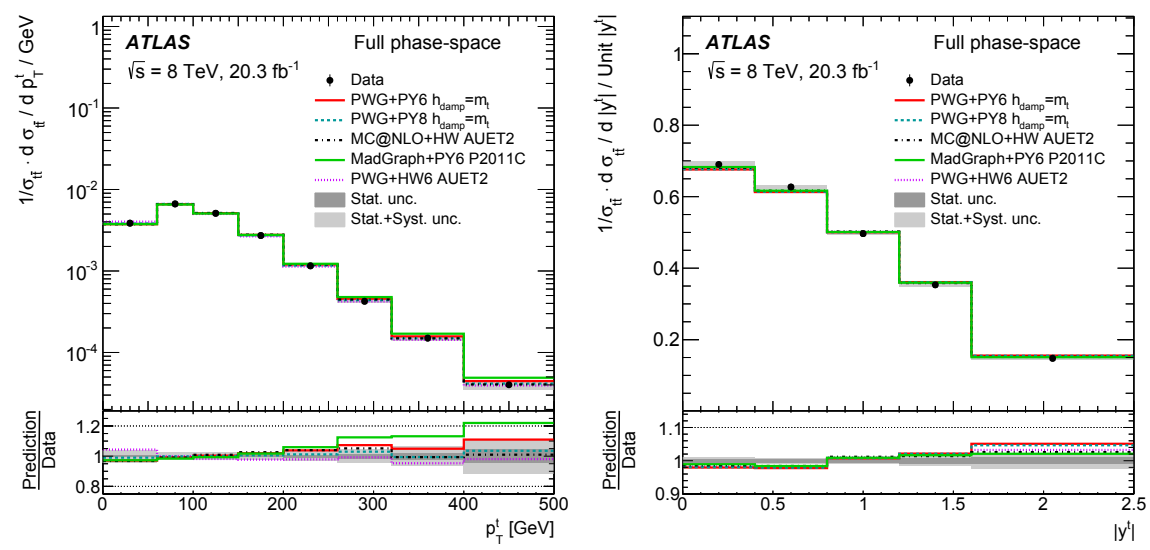

Figure 3: Full phase-space normalised differential cross-sections as a function of the transverse momentum, $p_{T}^{t}$, (left) and of the absolute value of the rapidity, $\left|y^{t, h a d}\right|$, (right). The lower part of the figure shows the ratio data to simulation. The grey bands indicate the total uncertainty on the data in each bin. The Powheg+PYTHIA generator with $h_{\text {damp }}=m_{t}$, which effectively regulates the high- $p_{T}$ radiation, and the CT10nlo PDF is used as the nominal prediction to correct for detector effects [5].

Table 1: List of $t \bar{t} W$ and $t \bar{t} Z$ decay modes and analysis channels targeting them [6].

\begin{tabular}{cccc}
\hline Process & $t \bar{t}$ decay & Boson decay & Channel \\
\hline \multirow{2}{*}{$t \bar{t} W^{ \pm}$} & $\left(\mu^{ \pm} v b\right)(q \bar{q} b)$ & $\mu^{ \pm} v$ & SS dimuon \\
& $\left(l^{ \pm} v b\right)\left(l^{\mp} v b\right)$ & $l^{ \pm} v$ & Trilepton \\
\hline \multirow{2}{*}{$t \bar{t} Z$} & $\left(\mu^{ \pm} v b\right)(q \bar{q} b)$ & $l^{+} l-$ & Trilepton \\
& $\left(l^{ \pm} v b\right)\left(l^{\mp} v b\right)$ & $l^{+} l^{-}$ & Tetralepton \\
\hline
\end{tabular}

\section{Trilepton analysis}

This region is divided into four signal regions with exactly three leptons. Three of these, out of which each requires an opposite-sign same flavour (OSSF) pair of leptons, whose mass is within $10 \mathrm{GeV}$ of the $\mathrm{Z}$ boson mass, are sensitive to $t \bar{t} Z$. The signal regions are categorised by their jet and b-jet multiplicities. The $3 l-Z-1 b 4 j$ region requires at least four jets, exactly one of which is b-tagged. The $3 l-Z-2 b 3 j$ region requires exactly three jets with at least two b-tagged jets. The $3 l-Z-2 \mathrm{~b} 4 \mathrm{j}$ region requires at least four jets, from which at least two are b-tagged and the $3 l$-noZ-2b region requires at least two and at most four jets, of which at least two jets are b-tagged. No OSSF lepton pair is allowed in the $\mathrm{Z}$ mass window. The sum of the lepton charges must be one. This region primarily targets the $t \bar{t} W$ process but has $t \bar{t} Z$ contribution comparable with that of the $3 l-Z-2 \mathrm{~b} 3 \mathrm{j}$ region. In addition to the signal regions, a control region is used to constrain the normalisation of the WZ background in data with exactly three leptons: $3 l$-WZ-CR.

\section{Tetralepton analysis}

The target process of this region is $t \bar{t} Z$, where both $\mathrm{W}$ bosons resulting from top-quark decays and the $\mathrm{Z}$ boson decay leptonically. Existence of two pairs of opposite-sign leptons is required, of which at least one pair having the same flavour. The lepton pair with opposite-sign same flavour and 
reconstructed invariant mass closest to the $\mathrm{Z}$ boson mass is attributed to the $\mathrm{Z}$ boson decay. According to the relative flavour of the other remaining two leptons, different flavour (DF) or same flavour (SF), and the number of b-tagged jets: one, or at least two $(1 \mathrm{~b}, 2 \mathrm{~b})$, four signal regions are defined: $4 l$-DF-1b, $4 l$-DF-2b, $4 l$-SF-1b and $4 l$-SF-2b. As in the trilepton analysis, a control region used to constrain the $\mathrm{ZZ}$ normalisation is defined to have exactly four reconstructed leptons: 4l-ZZ-CR.

The cross-sections for the two processes are determined by performing a simultaneous fit to the eight signal and two control regions defined above. The post-fit distributions for the expected yields
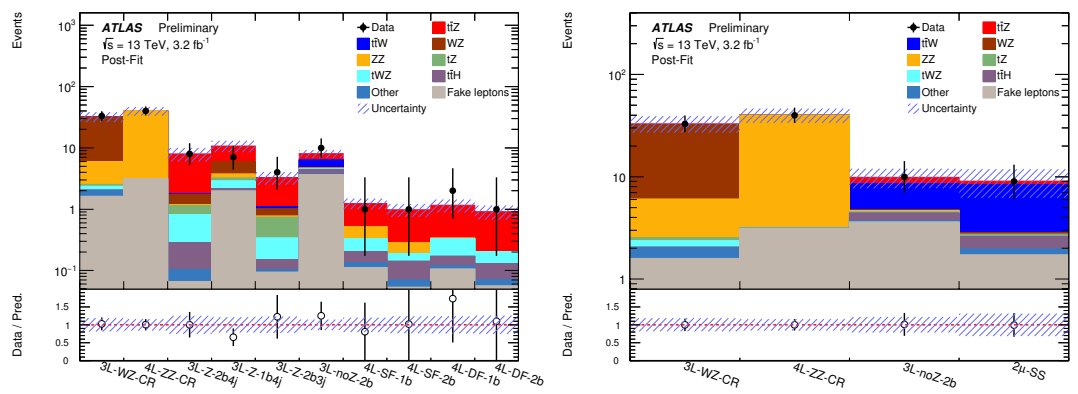

Figure 4: Expected yields after the fits. Data compared to signal and MC simulations for the $t \bar{t} Z$ (left) and $t \bar{t} W$ (right) fit in the relevant signal regions and the two control regions used to constrain the WZ and $\mathrm{ZZ}$ backgrounds. The lower part of the figure shows the ratio data to simulation. The hatched area corresponds to the total uncertainty on the predicted yields [6].

are presented in figure 4 . The determined cross-sections [6] are:

$$
\sigma_{t \bar{t} Z}=0.9 \pm 0.3 \mathrm{pb} \text { and } \sigma_{t \bar{t} W}=1.4 \pm 0.8 \mathrm{pb} .
$$

The theoretical predictions [6] to be compared with are:

$$
\sigma_{t \bar{t} Z}^{t h e o}=0.76 \pm 0.08 \mathrm{pb} \text { and } \sigma_{t \bar{t} W}^{t h e o}=0.57 \pm 0.06 \mathrm{pb} .
$$

\section{Single top-quark production}

The production measurement of the single top-quark is important to understand the properties of the $W t b$ vertex. In the leading-order perturbation theory, the single production of the top-quark is described by three subprocesses: the $t$-channel, the $W t$-channel and the $s$-channel. The subprocesses are distinguished by the virtuality of the exchanged $\mathrm{W}$ boson. This section presents an inclusive crosssection measurement in the $t$-channel at $\sqrt{s}=13 \mathrm{TeV}$ and in the $W t$ channel at $\sqrt{s}=8 \mathrm{TeV}$ as well as the first evidence of the single top-quark production in the $s$-channel at $8 \mathrm{TeV}$.

\subsection{Measurement of the inclusive cross-section of single top-quark $t$-channel production at $\sqrt{s}=13 \mathrm{TeV}$}

The single top-quark production in the $t$-channel is the dominant process of the channels mentioned above. It occurs by exchange of a virtual $\mathrm{W}$ boson between a light quark from one of the colliding protons and a $b$-quark from the other colliding proton. The cross-section of the single top-quark production induced by an up-quark is expected to be about twice the cross-section of the single-antiquark 
production induced by the down-quark since the density of the up-quark is twice the density of down-quark.

The measurement [8] is performed to determine the $t$-channel single top-quark production cross-section in the lepton+jets channel using $3.2 \mathrm{fb}^{-1}$ of pp collision data at a centre-of-mass energy of $13 \mathrm{TeV}$. For the measurement, events with an isolated muon and two jets with high transverse momenta are selected where one jet is required to pass the threshold of dedicated b-tagging algorithms. The discrimination between the signal and the background is achieved using a neural network $(\mathrm{NN})$. The most discriminating variables are found to be the reconstructed top-quark mass and the invariant mass of the two jets. Distributions of the two discriminating variables are shown in
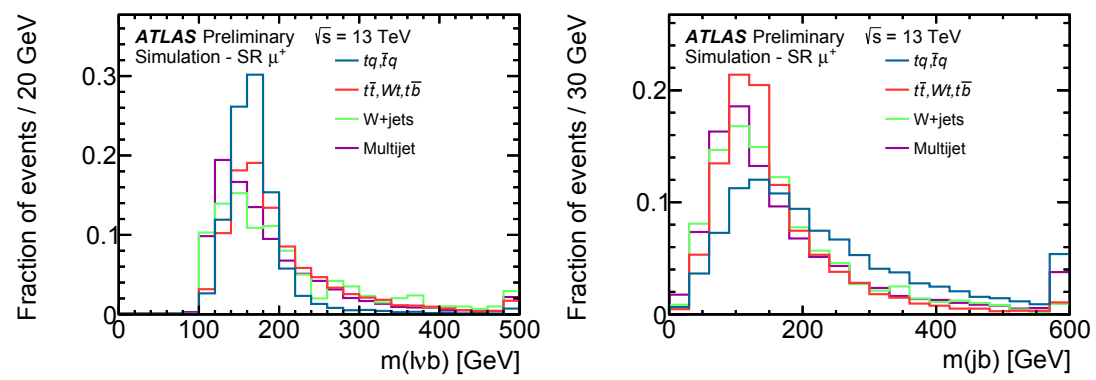

Figure 5: Distributions of the two most discriminating variables, the reconstructed top-quark mass (left) and the invariant mass of the jet pair (right) [8].

figure 5. The cross-section is measured by performing a binned maximum-likelihood (ML) fit to the NN output distribution. The cross-sections [8] determined are:

$$
\begin{gathered}
\sigma_{t q}=133 \pm 6 \text { (stat.) } \pm 24 \text { (syst.) } \pm 7 \text { (lumi) pb } \\
\sigma_{\bar{t} q}=96 \pm 5 \text { (stat.) } \pm 23 \text { (syst.) } \pm 5 \text { (lumi) pb. }
\end{gathered}
$$

\subsection{Measurement of the production cross-section of a single top-quark in association with a W boson at $\sqrt{s}=8 \mathrm{TeV}$}

While the other two channels of the single top-quark production have been observed at the Tevatron, the single top-quark production in association with a $\mathrm{W}$ boson has been observed for the first time at the LHC. Production of single top-quark in association with a $\mathrm{W}$ boson occurs via $b$-quark-induced partonic channels. This measurement [9] is performed with a data of $20.3 \mathrm{fb}^{-1}$ collected at $8 \mathrm{TeV}$ using dilepton events. The analysis requires two opposite-sign high $p_{T}$ leptons, missing transverse energy, one high $p_{T}$ jet which is required contain a $b$-hadron ( $b$-tagged). The separation of the signal from the background is performed through the use of boosted decision tree (BDT) algorithm. Each of the BDT combines a number of discriminating variables into one classifier. The training of the BDTs is done separately in three regions, 1 jet-1 tag, 2 jets-1 tag and 2jets-2tag using $W t$ (signal), and $t \bar{t}$ (main background) MC samples. The cross-section is then extracted by performing a profile likelihood fit to the classifier output distributions. Distributions of the boosted decision tree response for the three regions mentioned above are shown in figure 6. The measured cross-section [9] is:

$$
\left.\sigma_{W t}=23 \pm 1.3 \text { (stat. }\right)_{-3.5}^{+3.2} \text { (syst.) } \pm 1.1 \text { (lumi) pb, }
$$



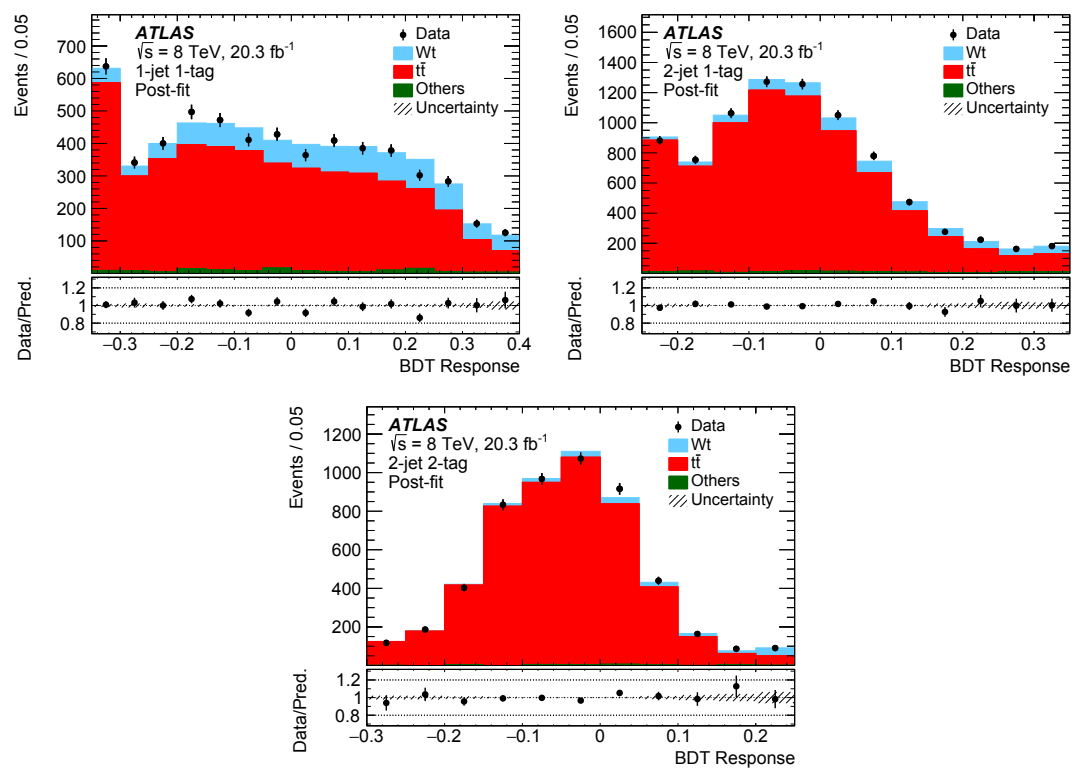

Figure 6: Boosted decision tree response for 1-jet 1-tag(upper left), 2-jet 1-tag (upper right) and 2-jet 2-tag (lower) events. Each contribution is normalised to its expectation. The lower part of the figure shows the ratio data to simulation. The hatched area represents the sum in quadrature of the statistical and systematic uncertainties [9].

where the theoretical prediction for the $W t$ production at NLO order with NNLL soft gluon corrections is $\sigma_{W t}^{\text {theo }}=22.37 \pm 1.52$ (stat.) $\mathrm{pb}[10]$.

\subsection{Evidence for single top-quark production in the s-channel in proton-proton collisions at $\sqrt{s}=8 \mathrm{TeV}$ using the Matrix Element Method}

The single top-quark production via $s$-channel is sensitive to new particles proposed by models of physics beyond the Standard Model such as charged Higgs boson or $W^{\prime}$ boson.

The measurement [11] is performed using $20.3 \mathrm{fb}^{-1}$ of data collected at the centre-of-mass energy of $8 \mathrm{TeV}$. Events with one isolated electron or muon, large missing transverse momentum and exactly two $b$-tagged jets are used. In addition to the signal region, two control regions are defined for the validation of $t \bar{t}$ and $W+$ jets backgrounds. Extraction of the signal is achieved by a maximum-likelihood fit to a discriminant. The discriminant is based on the matrix element method. The distribution of the matrix element discriminant for the $t \bar{t}$ validation region is shown in figure 7 . The result of the maximum-likelihood [11] fit is:

$$
\sigma_{W t}=4.8 \pm 0.8(\text { stat. })_{-1.3}^{+1.6} \text { (syst.) pb. }
$$

The result is in agreement with the Standard Model prediction. The observed significance of the result is 3.2 standard deviations, where the expected significance is 3.9 standard deviations. The post-fit distribution of the ME discriminant in the signal region is presented in figure 8 . 


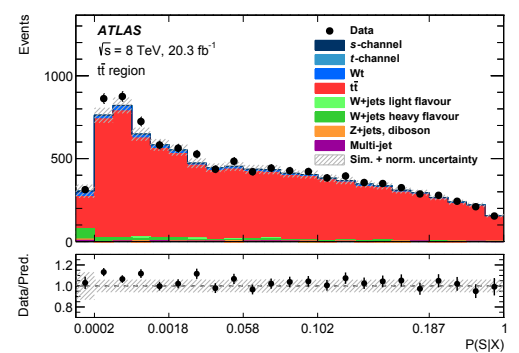

Figure 7: Matrix element discriminant for the $t \bar{t}$ validation region. Except for the data-driven multi-jet background all samples are scaled to their SM expectation. The lower part of the figure shows the ratio of data to simulation. The hatched bands represent the predicted normalisation uncertainty of all processes added in quadrature with the statistical uncertainty of the simulation [11].

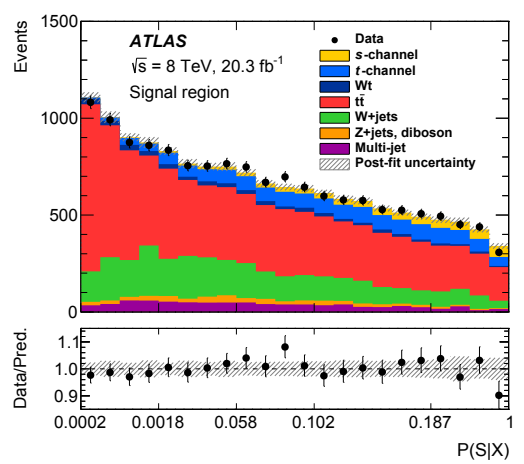

Figure 8: Post-fit distribution of the matrix element discriminant in the signal region. All samples are scaled by the fit result utilising all fit parameters. The lower part of the figure shows the ratio data to simulation. The hatched bands indicate the total uncertainty of the post-fit result including all correlations [11].

\section{References}

[1] CDF Collaboration, Phys.Rev.Lett., 74:2626-2631, 1995.

[2] D0 Collaboration, Phys.Rev.Lett., 74:2632-2637, 1995.

[3] ATLAS collaboration, JINST 3, S08003, 2008.

[4] ATLAS Collaboration, Phys. Lett. B761 (2016) 136.

[5] ATLAS Collaboration, arXiv:1511.04716v2 [hep-ex], 2016.

[6] ATLAS Collaboration, ATLAS-CONF-2016-003, 2015.

[7] ATLAS Collaboration, JHEP 1511 (2015) 172.

[8] ATLAS Collaboration, ATLAS-CONF-2015-079, 2015.

[9] ATLAS Collaboration, JHEP01 (2016) 064.

[10] N. Kidonakis Phys. Rev. D 82, 054018, 2010

[11] ATLAS Collaboration, Physics Letters B 756 (2016) 228-246. 